\title{
4D-STEM: Combining Pair Distribution Mapping and Multivariate Statistic Analysis to Quantify Structures in Complex Nanoscale Glasses
}

\author{
Xiaoke $\mathrm{Mu}^{1}$, Leyi Chen ${ }^{1}$ and Christian Kuebel $^{2}$ \\ ${ }^{1}$ Karlsruhe Institute of Technology, Germany, ${ }^{2}$ Karlsruhe Institute of Technology, Eggenstein-Leopoldshafen, \\ Baden-Wurttemberg, Germany
}

The detailed local structural characterization of amorphous materials has been a long-standing challenge for a fundamental understanding of glasses and glass composites as well as in the analysis of disordered organic materials. Information on the average short- and medium range order can be obtained by X-ray diffraction based pair distribution function (PDF) analysis. [1] More local information is accessible using electron diffraction based approaches. STEM diffraction mapping (4D-STEM) with a (sub)nanometer scale electron probe carries statistical information on the local atomic packing. Fluctuation electron microscopy has pioneered the interpretation of 4D-STEM datasets. It relies on a statistical analysis of the variations among diffraction profiles taken from different sample volumes to reveal structural inhomogeneities in terms of shortand medium-range order in amorphous materials [2]. An alternative way of using 4D-STEM explores the rotational symmetry of speckle diffraction patterns to investigate the local symmetry of amorphous structures [3].

We are combining electron diffraction based pair distribution analysis (ePDF) with 4D-STEM diffraction mapping to analyze the local atomic packing in nanoscale complex glasses [4] and polymer composites [5]. The ePDF describes the population of atomic pairs as a function of pair separation and, consequently, provides structural information on the short- and medium-range order (Figure 1). The data cube can be analyzed further by multivariate statistical analysis approaches, such as principal (PCA) and independent component analysis (ICA) [6], to reveal pure phase information and their distribution even in complex mixed compounds. In addition to providing information on the phase distribution, PDF mapping has the advantage to further reveal direct structural information, which can be interpreted in terms of the local atomic packing [7] and configuration [5].

With this presentation, we will introduce the methodology for STEM-PDF mapping and approaches to combine hyperspectral and statistical analysis [8] to get detail information on the local atomic packing in complex disordered materials. Application examples will range from metallic glass multilayers [4] (Figure 1) to complex nano glasses and structural variations in shear bands [7] to beam sensitive materials such as polymer composites in solar cells [5]. 
(a)

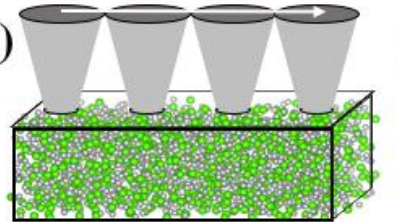

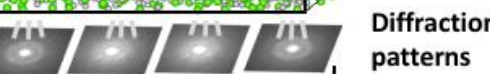


(e)

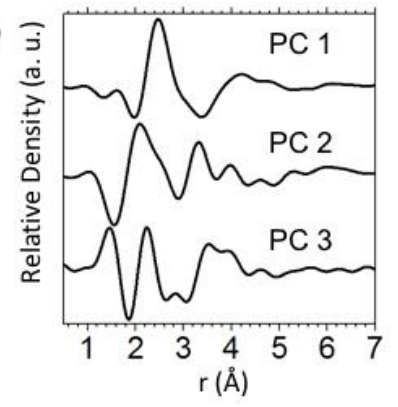

(f)

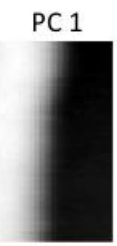

Scanned

e' beam

Sample

PDF cube
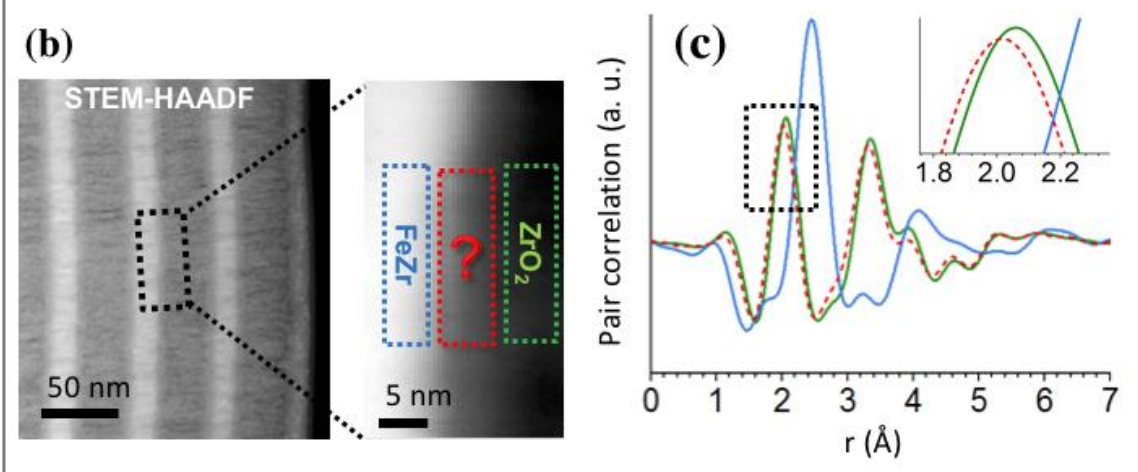

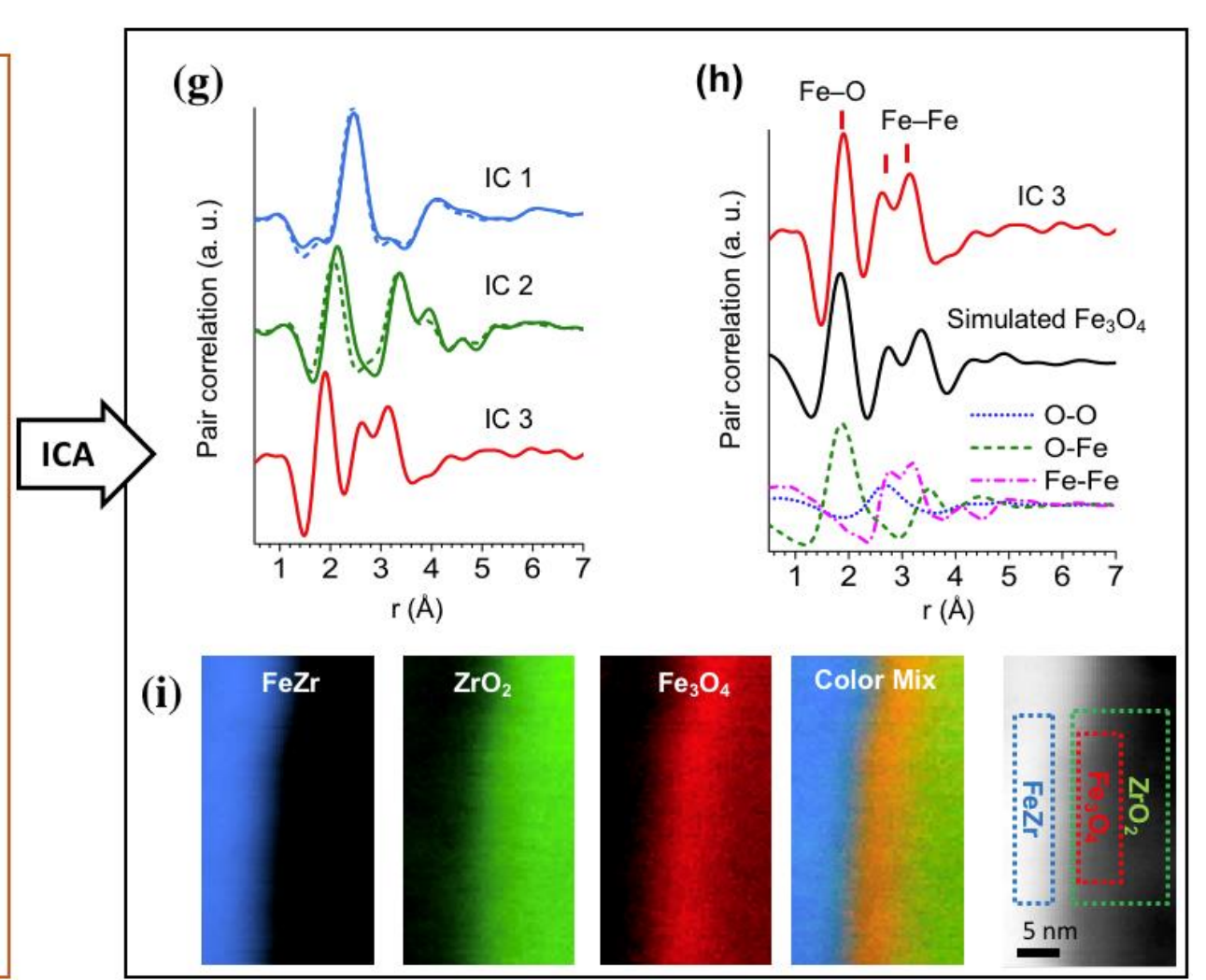

Figure 1. (a) Schematic depiction of STEM-PDF. (b) Conventional HAADF-STEM and virtual ADF-STEM image of an amorphous $\mathrm{ZrO} 2 / \mathrm{FeZr}$ multilayer with (c) the corresponding PDFs of the FeZr (blue) and the $\mathrm{ZrO} 2$ layer (green) and the interfacial region (red). PCA analysis: (d) scree plot, (e) first 3 PCs, and (f) their spatial distribution. (g) ICA analysis: ICs (solid lines) by ICA analysis of the PCA (dashed lines) results. (h) Comparison of IC3 (red) and a simulated PDF based on crystalline Fe3O4 (Marokite, black) and its partial PDFs. (i) Distribution map of the ICs (i.e. IC coefficients) and their correlation with the virtual ADF image. (reproduction from [8]). 

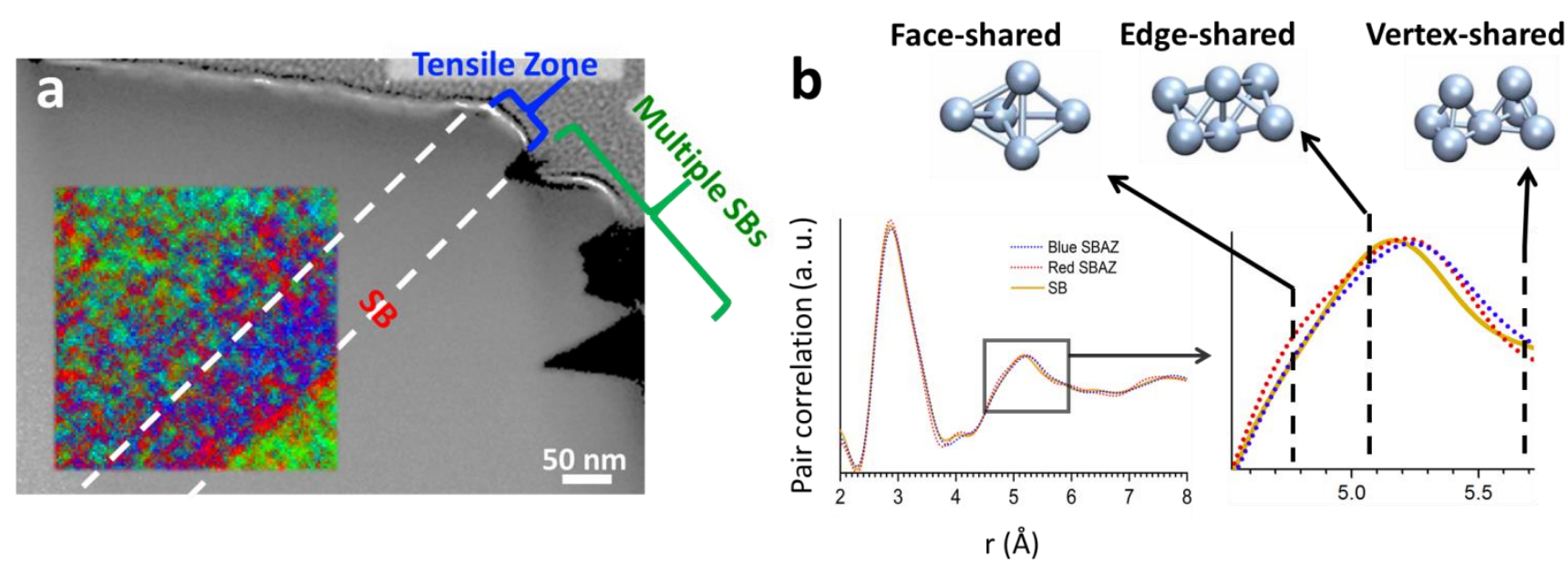

Figure 2. STEM-PDF analysis of a shear band (SB) and shear band affected zone (SBAZ): a) STEM-HAADF image superpositioned with PDF maps. Colors correspond to the region with reduction of geometrically favored motifs (red), Zr-rich clusters (Green) and Cu-rich clusters (blue). b) PDFs from the SBAZs and the $\mathrm{SB}$. The vertical dash lines indicate the features corresponding to face-, edge- and vertex-shared atomic connections.

\section{References}

[1] T. Egami, S. J. Billinge, Mater. Today. 6, 57 (2003)

[2] P. M. Voyles and D. A. Muller, Ultramicroscopy 93, 147 (2002).

[3] J. M. Gibson et al., Phys. Rev. Lett. 105, 1 (2010).

[4] X. Mu et al., Ultramicroscopy 168, 1 (2016).

[5] X. Mu et al., Microscopy, 68(4), 301-309, (2019).

[6] A. Hyvärinen and E. Oja, Neural Netw. 13, 411 (2000).

[7] X. Mu et al., Adv. Mater. (2021) 2007267.

[8] X. Mu et al., Acta Mat. (2021) submitted. 